\title{
IOT Based Smart Irrigation System
}

\author{
R.Muthukumar
}

Department of Electrical and Electronics Engineering

AMK Technological Polytechnic College, Sembarambakkam .India

\section{Abstract:}

This undertaking expects to build up a brilliant irrigation framework to screen the dampness and soil property of the irrigation land and runs the programmed control of the irrigation framework IoT remote units planned to be introduced in various irrigation zone and the control unit gets all the measure esteems from the remote units and after that keeps up when to water arrive and other control units and this framework progresses general control framework with its propelled observing structure.

\section{Introduction:}

Innovation is proceeding to improve our life and regularly in exceptional ways this time it is tied in with finding an answer for the unpleasant rider farming propelled advances have touched base to offer a successful answer for different irrigation issue. The web of thing is proceeding to advance offering more control over our living condition and permitting more straightforwardness in getting things done. Numerous consider this as the following huge skyline in the development of web and control application. The strong capacity of gathering, putting away, breaking down and appropriating information among various interface, applications, and gadgets are the fundamental favorable circumstances of IoT. We are this innovation IoT for soil observing and other irrigation tasks. Figure 1 and figure 2 demonstrates the square outline of the proposed strategy.

\section{Methodology:}

\section{Block Diagram}

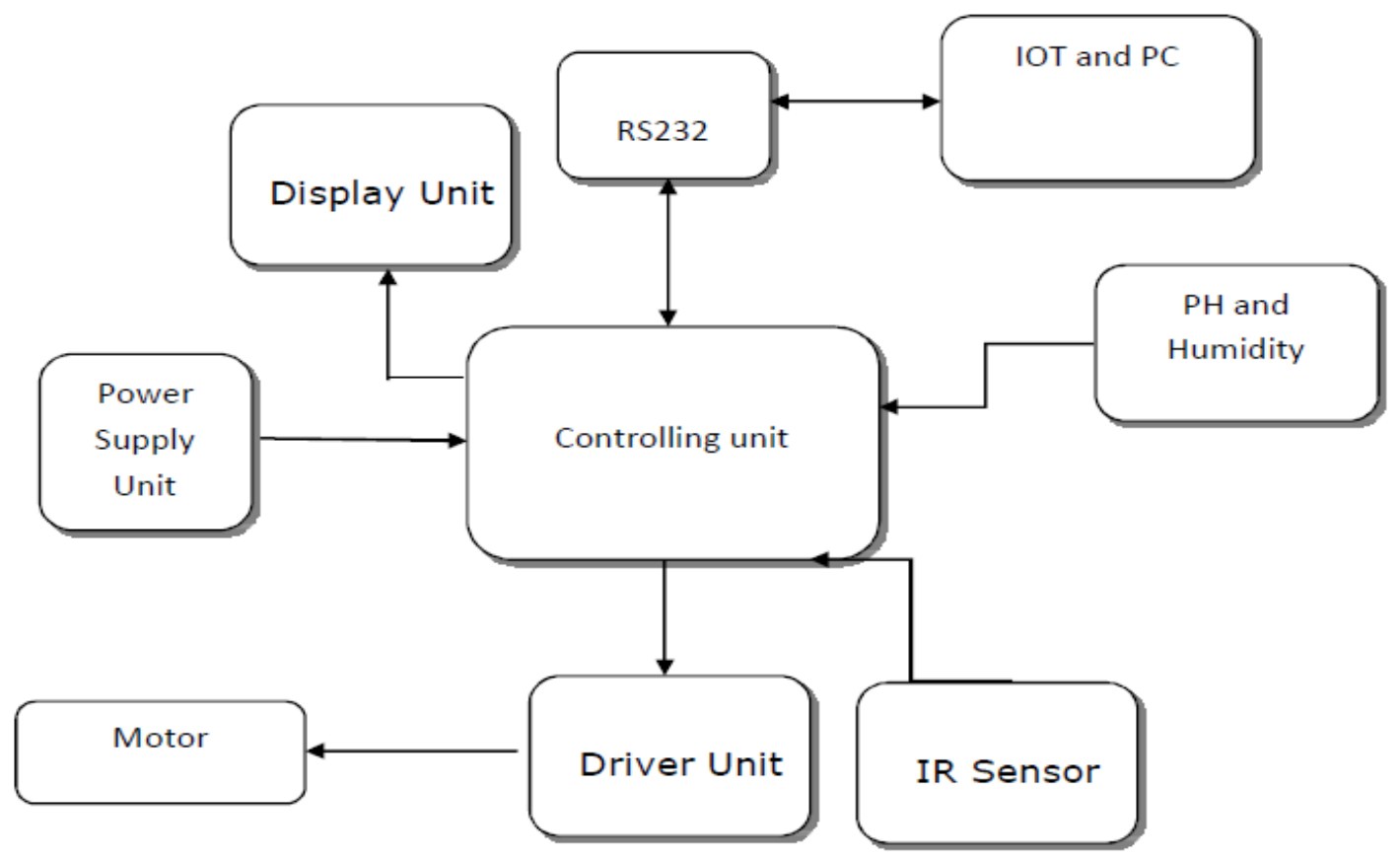

Figure 1: Crop Growing Section 


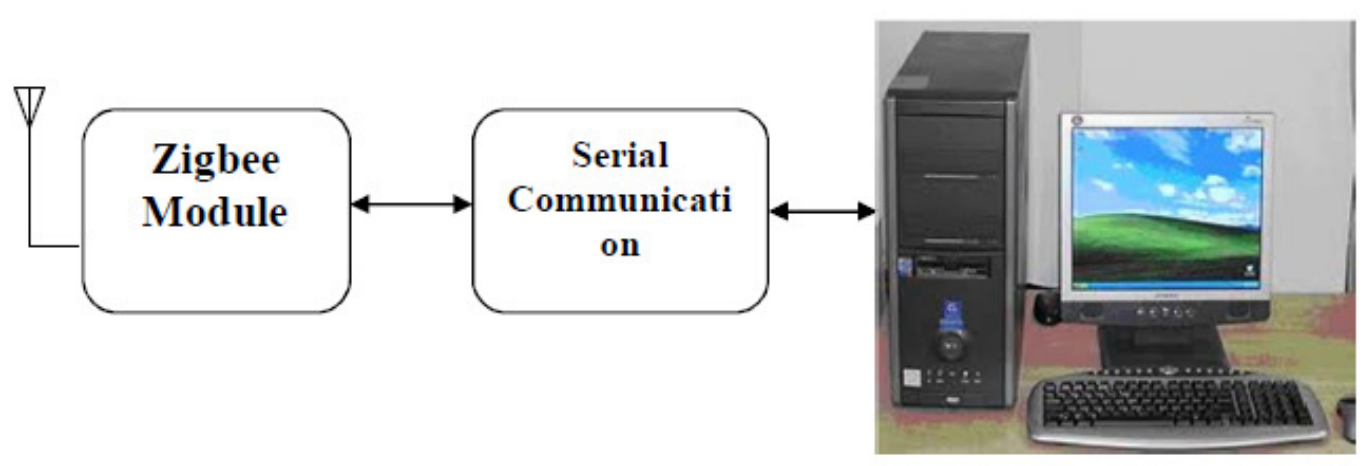

Figure 2: Monitoring Section

\section{EMBEDDED SYSTEM}

Implanted framework is a blend of equipment and programming used to accomplish a solitary errand. Installed framework is PC frameworks that screen, react to, or control an outer situation. Condition associated with framework through sensors, actuators and other I/O gadgets. Installed framework must meet planning and different compels forced on it by condition.

\section{VARIETY OF EMBEDDED SYSTEMS}

An inserted Router Board 112 with U.FL-RSMA braid and R52 smaller than expected PCI Wi-Fi card broadly utilized by remote Internet specialist co-ops (WISPs) in the Republic. Implanted frameworks traverse all parts of present day life and there are numerous cases of their utilization. Broadcast communications frameworks utilize various installed frameworks from phone switches for the system to cell phones toward the end-client. PC organizing utilizes committed switches and system scaffolds to course information. Customer hardware incorporate individual computerized colleagues (PDAs), mp3 players, cell phones, videogame reassures, advanced cameras, DVD players, GPS beneficiaries, and printers. Numerous family unit apparatuses, for example, microwaves, clothes washers and dishwashers, are including installed frameworks to give adaptability, proficiency and highlights. Progressed HVAC frameworks utilize organized indoor regulators to all the more precisely and effectively control temperature that can change by time of day and season. Home computerization utilizes wired-and remote systems administration that can be utilized to control lights, atmosphere, security, sound/visual, observation, and so on., all of which utilize implanted gadgets for detecting and controlling. Transportation frameworks from trip to vehicles progressively utilize implanted frameworks. New planes contain propelled flying, for example, inertial direction frameworks and GPS collectors that likewise have impressive security prerequisites. Different electric engines brushless DC engines, enlistment engines and DC engines are utilizing electric/electronic engine controllers. Autos, electric vehicles, and cross breed vehicles are progressively utilizing inserted frameworks to expand proficiency and diminish contamination. Other car wellbeing frameworks incorporate stopping automation (ABS), Electronic Stability Control (ESC/ESP), footing control (TCS) and programmed drive. Medicinal is proceeding to progress with more implanted frameworks for fundamental signs checking, electronic stethoscopes for increasing sounds, and different therapeutic imaging (PET, SPECT, CT, MRI) for non-intrusive inward reviews. 


\section{CHARACTERISTICS}

Installed frameworks are intended to do some particular assignment, instead of be a universally useful PC for numerous undertakings. Some likewise have continuous execution limitations that must be met, for reasons, for example, security and convenience; others may have low or no execution necessities, enabling the framework equipment to be improved to lessen costs.

Inserted frameworks are not generally independent gadgets. Numerous installed frameworks comprise of little, electronic parts inside a bigger gadget that fills a more broad need. For instance, the Gibson Robot Guitar includes an installed framework for tuning the strings, yet the general reason for the Robot Guitar is, obviously, to play music. Additionally, an inserted framework in a vehicle gives a particular capacity as a subsystem of the auto itself.

The program guidelines composed for implanted frameworks are alluded to as firmware, and are put away in read-just memory or Flash memory chips. They keep running with constrained PC equipment assets: little memory, little or non-existent console or screen.

\section{USER INTERFACE}

Installed frameworks extend from no UI at all committed just to one errand to complex graphical UIs that look like present day PC work area working frameworks. Straightforward inserted gadgets utilize catches, LEDs, realistic or character LCDs (for instance well known HD44780 LCD) with a basic framework. More refined gadgets which utilize a graphical screen with contact detecting or screen-edge catches give adaptability while limiting space utilized: the significance of the catches can change with the screen, and determination includes the characteristic conduct of pointing at what's coveted.

Handheld frameworks regularly have a screen with a "joystick catch" for a pointing gadget. A few frameworks furnish UI remotely with the assistance of a serial (e.g. RS-232, USB, $\mathrm{I}^{2} \mathrm{C}$, and so forth.) or system (e.g. Ethernet) association. This approach gives a few focal points: broadens the abilities of installed framework, evades the cost of a show, streamlines BSP, enables us to assemble rich UI on the PC. A decent case of this is the mix of an installed web server running on an implanted gadget, (for example, an IP camera) or a system switches. The UI is shown in an internet browser on a PC associated with the gadget, along these lines requiring no bespoke programming to be introduced.

\section{PROCESSORS IN EMBEDDED SYSTEMS}

Besides, installed processors can be broken into two general classes: normal microchips $(\mu \mathrm{P})$ and microcontrollers $(\mu \mathrm{C})$, which have numerous more peripherals on chip, diminishing expense and size. Differentiating to the PC and server advertises, a genuinely extensive number of fundamental CPU models are utilized; there are VonNeumann and different degrees of Harvard structures, RISC and in addition non-RISC and VLIW; word lengths fluctuate from 4-bit to 64-bits and past (for the most part in DSP processors) in spite of the fact that the most run of the mill remain 8/16-bit. Most engineering arrives in an expansive number of various variations and shapes, a significant number of which are additionally produced by a few distinct organizations.

\section{PIC 16F877A}

PIC microcontroller is the primary RISC based microcontroller manufactured in CMOS (corresponding metal oxide semiconductor) that utilizations isolate transport for direction and information permitting concurrent 
access of program and information memory. The primary preferred standpoint of CMOS and RISC mix is low power utilization bringing about a little chip measure with a little stick tally. The principle preferred standpoint of CMOS is that it has invulnerability to commotion than other creation strategies. Different microcontrollers offer various types of recollections. EEPROM, EPROM, FLASH and so on are a portion of the recollections of which FLASH is the most as of late created. Innovation that is utilized as a part of pic16F877A is streak innovation, with the goal that information is held notwithstanding when the power is turned off. Simple Programming and Erasing are different highlights of PIC 16F877A. Figure 3 speaks to stick chart of PIC 16F877A

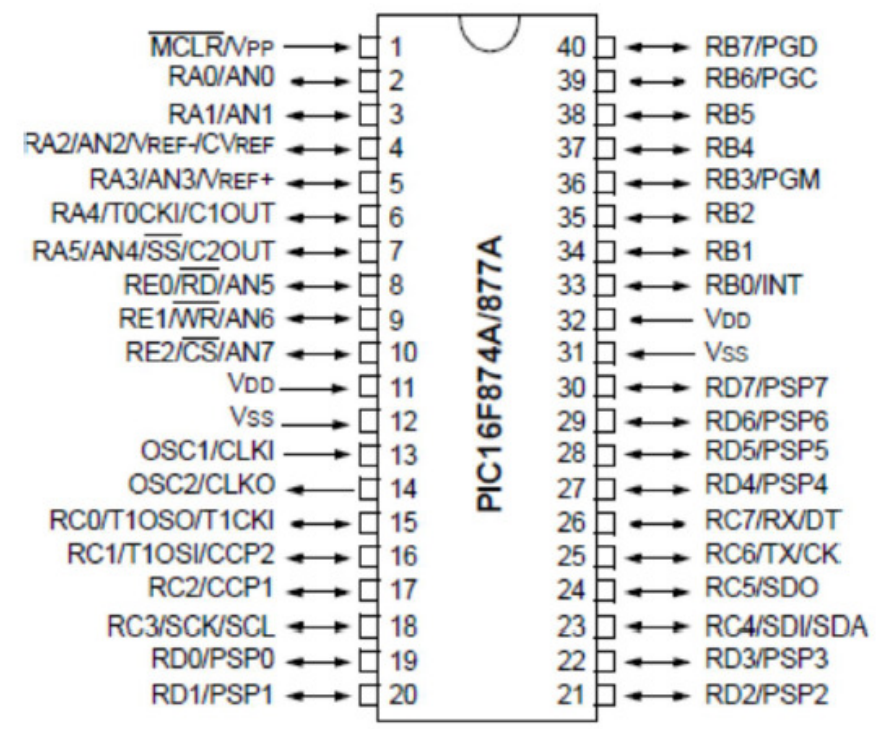

Figure 3: 16F877A Pin Diagram

\section{IR SENSOR}

IR transmitter and beneficiary LEDs have been around for quite a while so the innovation is as of now found in standard society (i.e. water features in washrooms/toilets/hand dryers). The Sharp IR Range Finder works by the procedure of triangulation. A beat of light (wavelength scope of $850 \mathrm{~nm}+/-70 \mathrm{~nm}$ ) is produced and afterward reflected back (or not reflected by any means). At the point when the light returns it returns at an edge that is subject to the separation of the reflecting item. Triangulation works by distinguishing this reflected pillar edge - by knowing the point, separation would then be able to be resolved as appeared underneath in figure

\section{4}

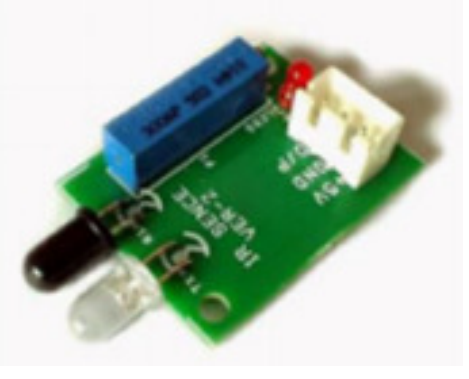

Figure 4: Infra red Sensor 
The IR run discoverer collector has an extraordinary exactness focal point that transmits the reflected light onto an encased direct CCD exhibit in light of the triangulation edge. The CCD cluster at that point decides the edge and makes the rangefinder at that point give a relating simple esteem that can be perused by a microcontroller. Extra to this, the Sharp IR Range Finder hardware applies a tweaked recurrence to the discharged IR bar. This going technique is relatively resistant to obstruction from encompassing light, and offers stunning lack of interest to the shade of the question being distinguished. At the end of the day, the sensor is equipped for recognizing a dark divider in full daylight with very nearly zero clamor.

\section{PH SENSOR}

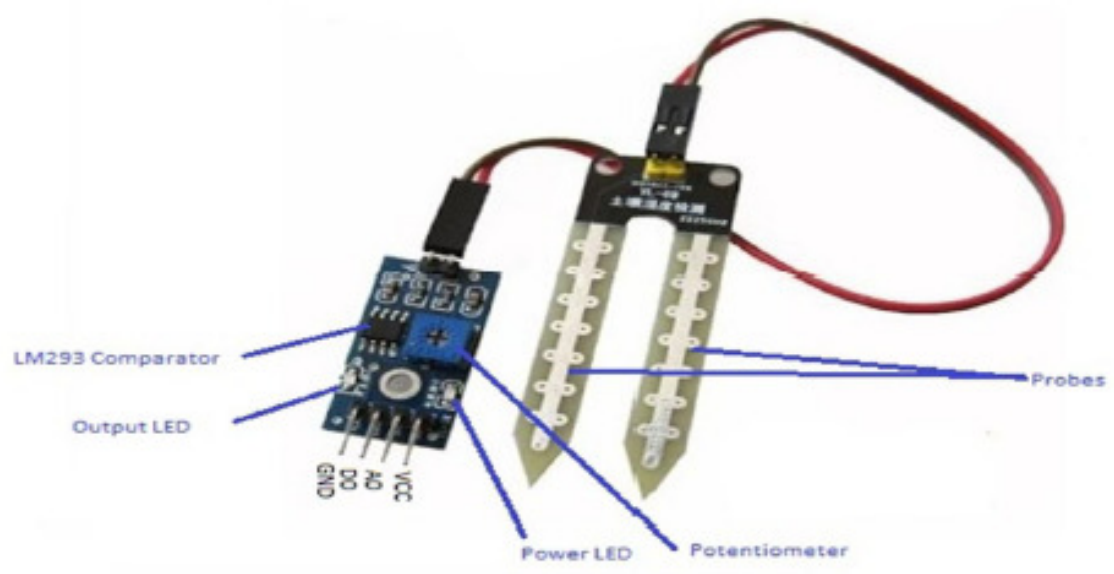

Figure 5 PH Sensor

The fundamental motivation behind a $\mathrm{pH}$ sensor is to keep up a $\mathrm{pH}$ level amid maturation that can advance the synergist transformation to liquor. A great $\mathrm{pH}$ meter measures the sharpness or alkalinity of a fluid. The $\mathrm{pH}$ meter includes the utilization of glass cathodes. Any $\mathrm{pH}$ meter should be adjusted before being utilized to test for the corrosive/alkalinity of a test arrangement. The $\mathrm{pH}$ meter requires customary adjustment with the goal that the glass anode creates an exact $\mathrm{pH}$ perusing.

\section{RELAY}

A relay is an electrical switch that uses an electromagnet to move the change from the off to on position rather than a man moving the switch. It takes a generally little measure of capacity to turn on a relay however the relay can control something that draws substantially more power. Ex: A relay is utilized to control the ventilation system in your home. The AC unit most likely keeps running off of 220VAC at around 30A. 
That is 6600 Watts! The curl that controls the relay may just need a couple of watts to pull the contacts

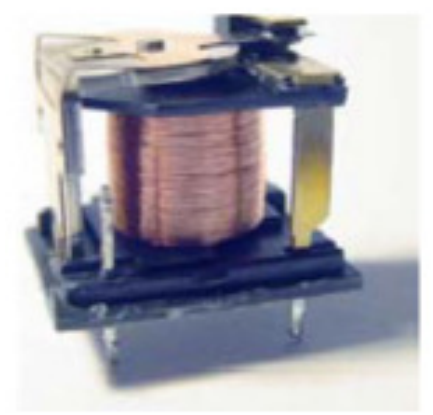

together.

Figure 6: Relay

\section{POWER SUPPLY}

There are numerous kinds of intensity supply. Most are intended to change over high voltage AC mains power to a reasonable low voltage supply for electronic circuits and different gadgets. A power supply can by separated into a progression of obstructs, every one of which plays out a specific capacity. For instance a $5 \mathrm{~V}$ directed supply:

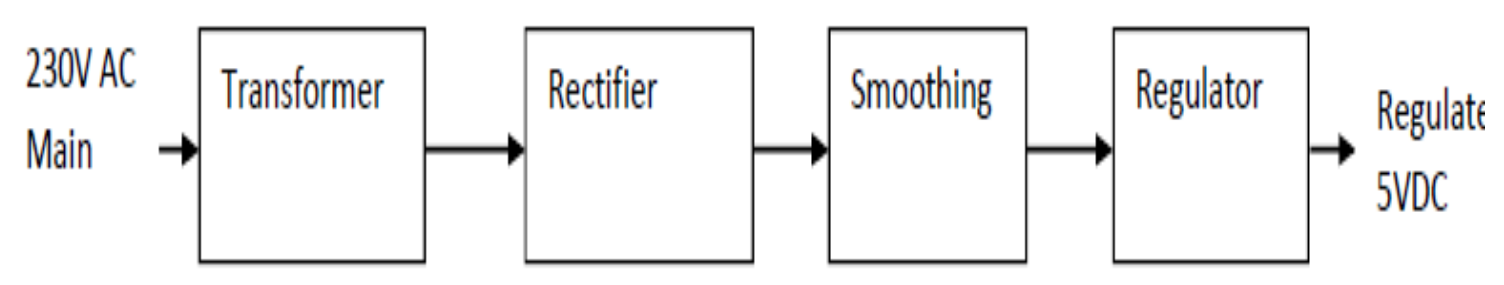

Figure 7: Power supply block Diagram

Every one of the squares is portrayed in more detail beneath:

- $\quad$ Transformer - ventures down high voltage AC mains to low voltage AC

- $\quad$ Rectifier - changes over AC to DC, yet the DC yield is shifting.

- $\quad$ Smoothing - smooth's the DC from fluctuating incredibly to a little swell.

- $\quad$ Regulator - wipes out swell by setting DC yield to a settled voltage

\section{Transformer}

The low voltage AC yield is reasonable for lights, radiators and uncommon AC engines. It isn't reasonable for electronic circuits except if they incorporate a rectifier and a smoothing capacitor. The changing DC yield is reasonable for lights, radiators and standard engines. It isn't appropriate for electronic circuits except if they incorporate a smoothing capacitor. The smooth DC yield has a little swell. 


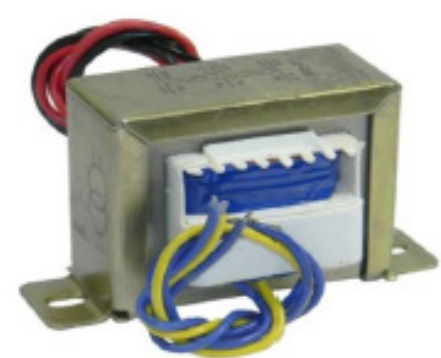

Figure 8: Transformer

\section{CIRCUIT DIAGRAM EXPLANATION}

The fundamental unit microcontroller and other supporting units are controlled from the power supply unit that gives directed $+5 \mathrm{v} 12 \mathrm{vdc}$. The LCD show that demonstrates the present status data of the task is associated with pins 19to22 and pins 27to30 of PIC16F877A microcontroller .The temperature sensor, stickiness sensor, IR sensor, PH sensor yield information are associated with pins 2, 4, 3, and 15 individually. The after effects of the undertaking and data are appeared in the pc and the pc is associated through RS232 serial interface and the relay unit is controlled through stick 16 of pic microcontroller

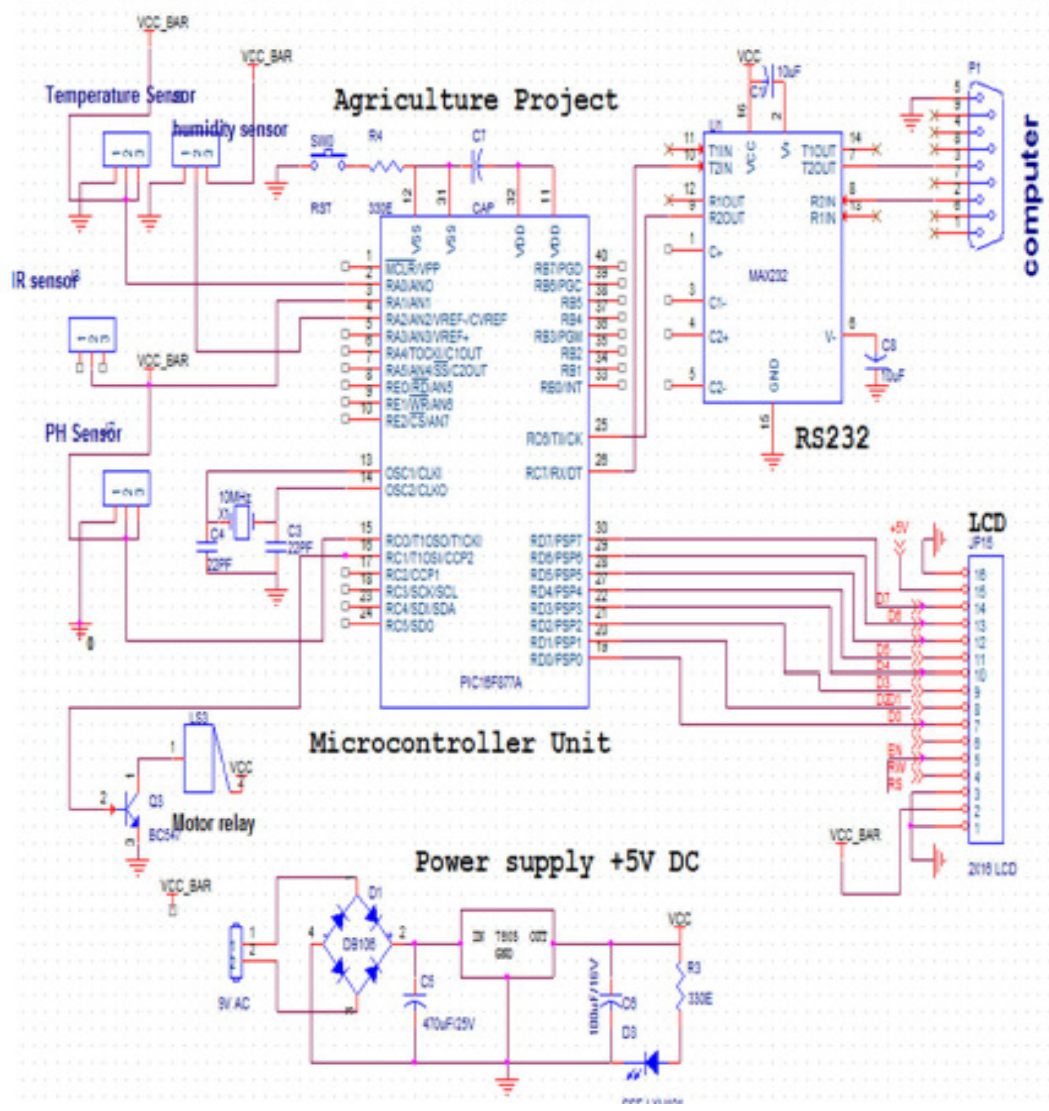

Figure 9: Circuit Diagram

\section{CONCLUSION}


We have effectively composed a remote control and checking framework for agriculturists that will graphically speak to the soil state of the harvest indirectly utilizing IoT. The composed framework is Powerproficient, financially savvy and easy to understand that is sufficiently productive to screen the yield condition and remotely control the peripherals of the irrigation framework which will make the activity of the agriculturists less demanding. This framework is versatile, as it enables any number of gadgets to be included with no real changes in its center engineering. Presently, the soil dampness, soil dampness sensor and $\mathrm{pH}$ sensor has been effectively interfaced with PIC16F877A. Remote transmission amongst sensor and facilitator hub is done through IoT. In future, the accompanying focuses will be secured. More sensors will be interfaced, for example, weight sensor to know climate conditions, Soil temperature sensor to make the framework considerably more productive. The product will be created on the server where a client can see the sensor information and timetable the irrigation plots remotely

\section{REFERENCE}

[1] W. A. Jury and HJ. Vaux, "The emerging global water crisis: Managing scarcity and conflict between water users", Adv. Agronomy, vol. 95, pp. 1-76, Sep. 2007.

[2] Mr.K.Kirubakaran, Selvi Ramlingam, S.Meerabai, V.Preethi, "Surveillance and Steering of Agricultural Field using Zigbee“, International Journal on Recent and Innovation Trends in Computing and Communication, vol 2, pp. 668 - 672, 2014. [3] Fan TongKe, "Smart Agriculture Based on Cloud Computing and IOT“'Journal of Convergence Information Technology, Vol. 8 ,Issue 2, pp.1,Jan2013.

[4] Khelifa Benahmed, Amel Douli, "Design of a New Smart-Irrigation System in the South of Algeria ", international conference IT4O, Tebessa, Algeria, 2014.

[5] D. Uckelmann, M. Harrison,F. Michahelles, “Architecting theInternet of Things“, Germany,Springer-Verlag Berlin Heidelberg, pp. 1-24, 2011.

[6] M.Weill, M.Souissi, "L’Internet des objets : concept ou réalité ? “, Réalités Industrielle,pp. 90-96, Novembre 2010.

[7] L.Atzori, A. Iera, G. Morabito, "The Internet of Things: A survey“, Science Direct Computer Networks 54 (2010), pp. 2787-2805, 2010.

[8] J. Gubbi, R. Buyya, S. Marusic, M.Palaniswami, "Internet of Things (IoT): A vision, architectural elements, and future directions, "SciVerse ScienceDirect, Future Generation Computer Systems 29 (2013), pp. 16451660, 2013.

[9] JP Vasseur, N. Agarwal, J. Hui, Z. Shelby, P. Bertrand, C. Chauvenet, "RPL: The IP routing protocol designed for lowpower and lossy networks“, Internet Protocol for Smart Objects (IPSO) Alliance, White Paper, April 2011. 